CAS-KITPC/ITP-022

\title{
A Note on Exact Solutions and Attractor Mechanism for Non-BPS Black Holes
}

\author{
Rong-Gen Cai ${ }^{\S \dagger}$, Da-Wei Pang ${ }^{\dagger \ddagger}$ \\ $\S$ Kavli Institute for Theoretical Physics China(KITPC) \\ at the Chinese Academy of Sciences \\ $\dagger$ Institute of Theoretical Physics, \\ Chinese Academy of Sciences \\ P.O.Box 2735, Beijing 100080, China \\ $\ddagger$ Graduate University of the Chinese Academy of Sciences \\ YuQuan Road 19A, Beijing 100049, China \\ cairg@itp.ac.cn, pangdw@itp.ac.cn
}

\begin{abstract}
We obtain two extremal, spherically symmetric, non-BPS black hole solutions to 4D supergravity, one of which carries D2-D6 charges and the other carries D0-D2-D4 charges. For the D2-D6 case, rather than solving the equations of motion directly, we assume the form of the solution and then find that the assumption satisfies the equations of motion and the constraint. Our D2-D6 solution is manifestly dual to the solution presented in 0710.4967. The D0-D2-D4 solution is obtained by performing certain $[S L(2, \mathbf{Z})]^{3}$ duality transformations on the D0-D4 solution in 0710.4967.
\end{abstract}




\section{Contents}

1 Introduction $\quad 1$

2 Preliminaries

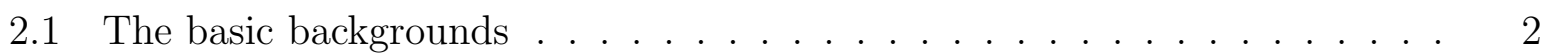

2.2 The STU model . . . . . . . . . . . . . . . . . . . . . . . . . . . . . . . . . . . . . .

2.3 The D0-D4 solution with complex moduli . . . . . . . . . . . 5

3 Exact Solution of the D2-D6 System 6

3.1 The solutions to the D2-D6 system with purely imaginary moduli . . . . 6

3.2 The solutions to the D2-D6 system with general complex moduli . . . . . 7

4 Adding D2 Charges to D0-D4 System $\quad 10$

4.1 U-duality for STU black holes . . . . . . . . . . . . . . . . . . . . . . . . . . . . .

4.2 The D0-D2-D4 solution . . . . . . . . . . . . . . . . 11

5 Summary and Discussion $\quad \lcm{14}$

\begin{tabular}{ll} 
References & 15 \\
\hline
\end{tabular}

\section{Introduction}

Black holes have provided a variety of interesting research subjects in recent years, one of which is the so-called "attractor mechanism". It means that, in certain black hole background, the moduli fields vary radially and "get attracted" to fixed values at the black hole horizon, which depend only on the quantized charges carried by the black hole. As a result, the entropy of the black hole is given only in terms of the charges and is independent of the asymptotic values of the moduli.

The attractor mechanism was firstly discovered in the mid 1990s in the context of $\mathrm{N}=2$ extremal black holes [1] and was generalized to higher derivative theories in [2]. The supersymmetric attractors were the main focus at first, but later it was realized that the attractor mechanism does not rely on supersymmetry in [3]. Non-supersymmetric attractors have been investigated extensively in recent years, see [4-8]. For reviews, see [9].

It has been found that the non-BPS attractors share many interesting properties with their BPS cousins, on condition that the non-BPS black holes are extremal. Although we can study the non-BPS attractors via the attractor equations, it is more useful to obtain the solution for the moduli fields in the whole space. However, it is rather complicated to obtain the exact solutions for non-BPS black holes because the equations of motion are second-order differential equations, rather than the first-order equations appearing in BPS cases. Usually people deal with this difficulty by making use of perturbative methods and numerical analysis, such as in [4] and related references. 
For STU black holes the situations have been better improved. It has been shown in $[10-13]$ that for the BPS case, we can find solutions to the equations of motion for the moduli, allowing us to obtain their values everywhere, by replacing the charges appeared in the attractive values of the moduli with the corresponding harmonic functions. A similar procedure has been carried out in [6] for non-BPS black holes carrying D2-D6 charges. However, the solutions were still limited in the sense that the moduli fields were taken to be purely imaginary.

Recently some interesting papers appeared [14] [15], which directly solved the equations of motion for the moduli in the STU model carrying D0-D4 charges. The moduli and charges were set to be equal in [14] while more general cases were discussed in [15]. Compared to the exact solutions in [6], their solutions for the moduli were complex.

We generalize the exact solutions in [14] and [15] to STU model carrying D2-D6 charges. It is difficult to solve the equations of motion directly because unlike the D0-D4 case, the superpotential contains a cubic term of the moduli fields. Instead of dealing with the first order flow equations, we try to find the solution to the second order equation of motion. By observing the exact solution in [6], which was manifestly dual to the existed D0-D4 system, we can assume that the D2-D6 solution is manifestly dual to the solution in [15] and check if it satisfies the equations of motion and the constraint. Fortunately, after a tedious calculation we find that the assumption is correct.

It has been known that the symplectic invariance of special geometry ensures that the Lagrangian has an $S p(8, \mathbf{Z})$ symmetry, which reduces to $[S L(2, \mathbf{Z})]^{3}$ at the level of the the equations of motion. Due to the $S L(2, \mathbf{Z})$ duality, we can obtain new solutions by making use of the "seed solution". We also obtain D0-D2-D4 solution from the D0-D4 solution obtained in [15] by some simple $S L(2, \mathbf{Z})$ duality transformations.

The rest of the note is organized as follows: In Section 2 we give a brief review of the attractor mechanism in STU model as well as the main procedures and results in [15]. Then we assume the form of the solution for STU model carrying D2-D6 charges with general complex moduli and find that the solution satisfies both the equations of motion and the constraint. Next we obtain D0-D2-D4 solution by dualizing the D0-D4 solution in $[15]$ under $[S L(2, \mathbf{Z})]^{3}$ symmetry. We summarize the results and discuss some related topics in the final section.

\section{Preliminaries}

\subsection{The basic backgrounds}

Type IIA string theory compactified on a $C Y_{3}$ manifold gives $\mathcal{N}=2$ supersymmetry. The moduli fields belong to the vector multiplets and hypermultiplets of the resulting lowenergy effective theory. The moduli in the vector multiplets are fixed according to the attractor mechanism while the moduli in the hypermultiplets play no role in the attractor mechanism. The low-energy dynamics for the vector multiplets is completely determined by a prepotential. If the Calabi-Yau manifold has $h(1,1)=N$, we have $N$ vector multiplets 
and $N+1$ gauge fields, where the additional gauge field is the graviphoton coming from the gravity multiplet. The leading order prepotential, ignoring any $\alpha^{\prime}$ corrections, is given as

$$
F=D_{A B C} \frac{X^{A} X^{B} X^{C}}{X^{0}}
$$

where $A, B, C=1, \cdots, N$. The intersection number $D_{a b c}$ are defined as

$$
6 D_{A B C}=\int_{C Y_{3}} \alpha_{A} \wedge \alpha_{B} \wedge \alpha_{C},
$$

where $\alpha_{A}$ denote the integer basis for $H^{2}\left(C Y_{3}, \mathbf{Z}\right)$.

Type IIA string theory admit D0, D2, D4 and D6 branes. D0 and D6 branes are electrically and magnetically charged with respect to the graviphoton, while D2 and D4 branes are electrically and magnetically charged with respect to the other $N$ gauge fields. One can express the charge configuration collectively as $\left(q_{0}, q_{A}, p^{0}, p^{A}\right)$, with $A=1,2, \cdots, N$. To be more precise, let $\Sigma^{A}$ be a basis of 4-cycles dual to $\alpha_{A}$ introduced above, and let $\hat{\Sigma}_{A}$ be a basis dual to $\Sigma^{A}$. Then the magnetic charge carried by the $\mathrm{D} 4$ brane wrapping $\Sigma^{A}$ is $p^{A}$, while the electric charge carried by the brane wrapping $\hat{\Sigma}_{A}$ is $q_{A}$.

The Kähler potential is given by

$$
K=-\ln \left[i \sum_{\Lambda=0}^{N}\left(\bar{X}^{\Lambda} F_{\Lambda}-X^{\Lambda} \bar{F}_{\Lambda}\right)\right]
$$

where $F_{\Lambda} \equiv \partial_{\Lambda} F$. If we choose the inhomogeneous coordinates $z^{\lambda}=\frac{X^{\Lambda}}{X^{0}}$ and set the gauge $X^{0}=1$, the Kähler potential becomes

$$
K=-\ln \left[-i D_{A B C}\left(z^{A}-\bar{z}^{A}\right)\left(z^{B}-\bar{z}^{B}\right)\left(z^{C}-\bar{z}^{C}\right)\right] .
$$

The superpotential is given by

$$
W=q_{\Lambda} X^{\Lambda}-p^{\Lambda} F_{\Lambda}
$$

Here in the $X^{0}=1$ gauge the superpotential becomes

$$
W=q_{0}+q_{A} z^{A}-3 D_{A B C} z^{A} z^{B} p^{C}+p^{0} D_{A B C} z^{A} z^{B} z^{C} .
$$

\subsection{The STU model}

Now let us concentrate on the so-called STU model, which can be interpreted in terms of type IIA string theory on a $T^{6}$ of the form $T^{2} \times T^{2} \times T^{2}$. This model contains three vectormultiplets, i.e. $N=3$. The prepotential and the Kähler potential can be obtained immediately.

$$
F=\frac{X^{1} X^{2} X^{3}}{X^{0}}
$$




$$
K=-\ln \left[-i\left(z^{1}-\bar{z}^{1}\right)\left(z^{2}-\bar{z}^{2}\right)\left(z^{3}-\bar{z}^{3}\right)\right]=-\ln \left(8 y^{1} y^{2} y^{3}\right),
$$

where we have rewritten $z^{a}=x^{a}-i y^{a}(a=1,2,3)$ for later convenience. The metric and connection on the moduli space are given by

$$
G_{a \bar{b}}=-\frac{\delta_{a b}}{\left(z^{a}-\bar{z}^{a}\right)^{2}}=\frac{\delta_{a b}}{\left(2 y^{a}\right)^{2}}, \quad G^{a \bar{b}}=-\delta^{a b}\left(z^{a}-\bar{z}^{a}\right)^{2}=\delta^{a b}\left(2 y^{a}\right)^{2}, \quad \Gamma_{a a}^{a}=-\frac{2}{z^{a}-\bar{z}^{a}} .
$$

The superpotential can be written as

$$
W=q_{0}+q_{a} z^{a}-p^{1} z^{2} z^{3}-p^{2} z^{1} z^{3}-p^{3} z^{1} z^{2}+p^{0} z^{1} z^{2} z^{3} .
$$

Consider a static, spherically symmetric four-dimensional spacetime with the metric

$$
d s^{2}=-e^{2 U(\tau)} d t^{2}+e^{-2 U(\tau)} d \vec{x}^{2},
$$

where $\tau=1 /|\vec{x}|$. Note that the horizon locates at $\tau=0$ and the asymptotic infinity tends to $\tau \rightarrow \infty$. The effective Lagrangian describing the system is

$$
\mathcal{L}_{e f f}=\dot{U}^{2}+G_{a \bar{b}} \dot{z}^{a} \dot{\bar{z}}^{\bar{b}}+e^{2 U} V_{\mathrm{BH}},
$$

where the dot denotes differentiation with respect to $\tau$. The term $V_{\mathrm{BH}}$ in the above effective Lagrangian is the so-called "effective potential", which is given by

$$
V_{B H}=|D Z|^{2}+|Z|^{2}=G^{a \bar{b}}\left(D_{a} Z\right)\left(\bar{D}_{\bar{b}} \bar{Z}\right)+Z \bar{Z} .
$$

$Z$ is the central charge of the SUSY algebra and is expressed as $Z=e^{K / 2} W$ in our case. The covariant derivative of the central charge is

$$
D_{a} Z=e^{K / 2}\left[\partial_{a}+\left(\partial_{a} K\right)\right] W .
$$

One can obtain the equations of motion by varying the above effective Lagrangian.

$$
\begin{gathered}
\ddot{U}=e^{2 U} V_{B H}, \\
\ddot{z}^{a}+\Gamma_{b c}^{a} \dot{z}^{b} \dot{z}^{c}=e^{2 U} G^{a \bar{b}} \partial_{\bar{b}} V_{\mathrm{BH}} .
\end{gathered}
$$

In addition, there is a Hamiltonian constraint on the solutions

$$
\dot{U}^{2}+G_{a \bar{b}} \dot{z}^{a} \dot{\bar{z}}^{\bar{b}}-e^{2 U} V_{B H}=c^{2},
$$

where $c^{2}=0$ for extremal black holes.

When the black hole in the solution is extremal, the values of the moduli $z^{a}$ will be fixed at the horizon, irrespective of their values at asymptotic infinity. The attractor values can be obtained by minimizing the effective potential, either directly or via the attractor equations. The entropy of the extremal black hole, whether BPS or not, is given by the effective potential evaluated at the extremum:

$$
S=\frac{A}{4}=\left.\pi V_{\mathrm{BH}}\right|_{\mathrm{ext}} .
$$




\subsection{The D0-D4 solution with complex moduli}

The simplest solution of the D0-D4 system is the case of a D0-D4-D4-D4 black hole without B-fields, where $q_{0}>0$ and $p^{a}>0$ but $p^{0}=q_{a}=0$, which results in a BPS configuration. The solution to the effective Lagrangian is

$$
\begin{gathered}
e^{-4 U}=4 H_{0} H^{1} H^{2} H^{3}, \\
z^{a}=-i \sqrt{\frac{2 H_{0} H^{a}}{s_{a b c} H^{b} H^{c}}},
\end{gathered}
$$

where $s_{a b c}=\left|\epsilon_{a b c}\right|$ and the harmonic functions are given as follows

$$
H^{a}=\frac{1}{\sqrt{2}}+p^{a} \tau, \quad H_{0}=\frac{1}{\sqrt{2}}+q_{0} \tau .
$$

The attractor values of the moduli become

$$
z^{a}=-i \sqrt{\frac{2 q_{0} p^{a}}{s_{a b c} p^{b} p^{c}}} .
$$

One can obtain a non-BPS solution by simply analytic continuation from the BPS case, that is, we assume $q_{0}<0, p^{a}>0$. Thus the harmonic functions turn out to be

$$
H^{a}=\frac{1}{\sqrt{2}}+p^{a} \tau, \quad H_{0}=-\frac{1}{\sqrt{2}}+q_{0} \tau
$$

and we can write down the solution

$$
\begin{gathered}
e^{-4 U}=\left|4 H_{0} H^{1} H^{2} H^{3}\right|, \\
z^{a}=-i \sqrt{\frac{-2 H_{0} H^{a}}{s_{a b c} H^{b} H^{c}}} .
\end{gathered}
$$

The attractor values of the moduli

$$
z^{a}=-i \sqrt{\frac{-2 q_{0} p^{a}}{s_{a b c} p^{b} p^{c}}} .
$$

The authors of [15] generalized the simple non-BPS solution to situations where the asymptotic moduli are more general and/or there are more charges present. In particular, they normalized the asymptotic volume moduli so that $\left.y^{a}\right|_{\infty}=1$ but kept the asymptotic B-fields $x_{\infty}^{a}=B^{a}$ as free variables. They obtained the following solution by solving the equations of motion directly,

$$
\begin{gathered}
e^{-4 U}=-4 H_{0} H^{1} H^{2} H^{3}-B^{2}, \\
z^{a}=\frac{B-i e^{-2 U}}{s_{a b c} H^{b} H^{c}},
\end{gathered}
$$

where the harmonic functions are given by

$$
H^{a}=\frac{1}{\sqrt{2}}+p^{a} \tau, \quad H_{0}=-\frac{1}{\sqrt{2}}\left(1+B^{2}\right)+q_{0} \tau .
$$




\section{Exact Solution of the D2-D6 System}

The exact solutions to the D0-D4 system with general complex moduli were obtained in [14] and [15] by solving the equations of motion directly. However, it would be more difficult to carry out similar procedures for the D2-D6 system due to the cubic term involving the D6 charge $p^{0}$ in the superpotential. However, the exact solutions to the D2-D6 system with purely imaginary moduli were obtained in [6]. The basic idea was that one could take the horizon values of the moduli and replace the charges with corresponding harmonic functions. Then one could check if the ansatz satisfies the equations of motion as well as the constraint. Fortunately after a somewhat more involved calculation one found that the ansatz satisfied all the requirements. The result was manifestly dual to the known D0-D4 system.

Inspired by such a method, we make a similar ansatz for the moduli of the D2-D6 system and find that the ansatz also satisfies the equations of motion and the constraint. Furthermore, the solution to the D2-D6 system is also manifestly dual to the solution obtained in [15].

\subsection{The solutions to the D2-D6 system with purely imaginary moduli}

In this subsection we will list the main results of [6], which leads to our ansatz. The superpotential for the D2-D6 system is

$$
W=q_{a} z^{a}+p^{0} z^{1} z^{2} z^{3} .
$$

If $p^{0} q_{1} q_{2} q_{3}<0$, the resulting configuration is BPS, while $p^{0} q_{1} q_{2} q_{3}>0$ corresponds to nonBPS configuration. The attractor values for the moduli in the non-BPS case are given by

$$
z^{1}=-i \sqrt{\frac{q_{2} q_{3}}{p^{0} q_{1}}}, \quad z^{2}=-i \sqrt{\frac{q_{1} q_{3}}{p^{0} q_{2}}}, \quad z^{3}=-i \sqrt{\frac{q_{1} q_{2}}{p^{0} q_{3}}} .
$$

As pointed out in [6], for both BPS and non-BPS STU black holes, we can find solutions to the equations of motion for the moduli, allowing us to obtain their values everywhere. Such solutions can be obtained by replacing the charges in the attractor values of the moduli with the corresponding harmonic functions. Furthermore, we have to check that if such solutions satisfy the equations of motion and the constraint.

For the D2-D6 case, after replacing the charges with harmonic functions, the solutions turn out to be

$$
\begin{gathered}
e^{-2 U}=2 \sqrt{H^{0} H_{1} H_{2} H_{3}} \\
z^{1}=-i \sqrt{\frac{H_{2} H_{3}}{H^{0} H_{1}}}, \quad z^{2}=-i \sqrt{\frac{H_{1} H_{3}}{H^{0} H_{2}}}, \quad z^{3}=-i \sqrt{\frac{H_{1} H_{2}}{H^{0} H_{3}}} .
\end{gathered}
$$

Note that the above equations can also be expressed as

$$
z^{1}=-i \frac{e^{-2 U}}{2 H^{0} H_{1}}, \quad z^{2}=-i \frac{e^{-2 U}}{2 H^{0} H_{2}}, \quad z^{3}=-i \frac{e^{-2 U}}{2 H^{0} H_{3}} .
$$


They proved that such an ansatz did satisfy the equations of motion and the constraint by working out the terms in these equations explicitly.

\subsection{The solutions to the D2-D6 system with general complex moduli}

In this subsection we will show that our ansatz satisfies the equations of motion and the

constraint of the D2-D6 system. Furthermore, the exact solution is also manifestly dual to the solution obtained in [15]. We would like to set $z^{a}=x^{a}-i y^{a}$ for convenience.

The effective Lagrangian becomes

$$
\begin{aligned}
\mathcal{L}_{\text {eff }} & =(\dot{U})^{2}+G_{a \bar{b}} \dot{z}^{a} \dot{\bar{z}}^{\bar{b}}+e^{2 U} V_{\mathrm{BH}} \\
& =(\dot{U})^{2}+\frac{1}{4} \sum_{a=1}^{3} \frac{\left(\dot{x}^{a}\right)^{2}+\left(\dot{y}^{a}\right)^{2}}{\left(y^{a}\right)^{2}}+e^{2 U} V_{\mathrm{BH}} .
\end{aligned}
$$

The expression for the effective potential is given by

$$
\begin{aligned}
V_{\mathrm{BH}} & =|D Z|^{2}+|Z|^{2} \\
& =G^{a \bar{b}} D_{a} Z \bar{D}_{\bar{b}} \bar{Z}+Z \bar{Z} \\
& =G^{a \bar{b}}\left(e^{K / 2}\left(\partial_{a}+\partial_{a} K\right) W\right)\left(\overline{e^{K / 2}\left(\partial_{b}+\partial_{b} K\right) W}\right)+e^{K} W \bar{W} .
\end{aligned}
$$

We can obtain the following explicit expression for the effective potential by making use of (2.8), (2.9) and (3.1),

$$
\begin{aligned}
V_{\mathrm{BH}}= & \frac{1}{2 y^{1} y^{2} y^{3}}\left\{q_{1}^{2}\left[\left(x^{1}\right)^{2}+\left(y^{1}\right)^{2}\right]+q_{2}^{2}\left[\left(x^{2}\right)^{2}+\left(y^{2}\right)^{2}\right]+q_{3}^{2}\left[\left(x^{3}\right)^{2}+\left(y^{3}\right)^{2}\right]\right. \\
& +\left(p^{0}\right)^{2}\left[\left(x^{1}\right)^{2}+\left(y^{1}\right)^{2}\right]\left[\left(x^{2}\right)^{2}+\left(y^{2}\right)^{2}\right]\left[\left(x^{3}\right)^{2}+\left(y^{3}\right)^{2}\right]+2 p^{0} q_{1} x^{2} x^{3}\left[\left(x^{1}\right)^{2}+\left(y^{1}\right)^{2}\right] \\
& +2 p^{0} q_{2} x^{1} x^{3}\left[\left(x^{2}\right)^{2}+\left(y^{2}\right)^{2}\right]+2 p^{0} q_{3} x^{1} x^{2}\left[\left(x^{3}\right)^{2}+\left(y^{3}\right)^{2}\right] \\
& \left.+2 q_{1} q_{2} x^{1} x^{2}+2 q_{1} q_{3} x^{1} x^{3}+2 q_{2} q_{3} x^{2} x^{3}\right\} .
\end{aligned}
$$

Then the equations of motion and the constraint turn out to be

$$
\ddot{U}=e^{2 U} V_{\mathrm{BH}},
$$

$$
\begin{aligned}
& \frac{1}{2} \frac{d}{d \tau}\left[\frac{\dot{x}^{1}}{\left(y^{1}\right)^{2}}\right]=e^{2 U} \frac{\partial V_{\mathrm{BH}}}{\partial x^{1}}, \\
& \frac{1}{2} \frac{d}{d \tau}\left[\frac{\dot{x}^{2}}{\left(y^{2}\right)^{2}}\right]=e^{2 U} \frac{\partial V_{\mathrm{BH}}}{\partial x^{2}} \\
& \frac{1}{2} \frac{d}{d \tau}\left[\frac{\dot{x}^{3}}{\left(y^{3}\right)^{2}}\right]=e^{2 U} \frac{\partial V_{\mathrm{BH}}}{\partial x^{3}}
\end{aligned}
$$




$$
\begin{aligned}
& \frac{1}{2} \frac{d}{d \tau}\left[\frac{\dot{y}^{1}}{\left(y^{1}\right)^{2}}\right]+\frac{1}{2\left(y^{1}\right)^{3}}\left[\left(\dot{x}^{1}\right)^{2}+\left(\dot{y}^{1}\right)^{2}\right]=e^{2 U} \frac{\partial V_{\mathrm{BH}}}{\partial y^{1}}, \\
& \frac{1}{2} \frac{d}{d \tau}\left[\frac{\dot{y}^{2}}{\left(y^{2}\right)^{2}}\right]+\frac{1}{2\left(y^{2}\right)^{3}}\left[\left(\dot{x}^{2}\right)^{2}+\left(\dot{y}^{2}\right)^{2}\right]=e^{2 U} \frac{\partial V_{\mathrm{BH}}}{\partial y^{2}} \text {, } \\
& \frac{1}{2} \frac{d}{d \tau}\left[\frac{\dot{y}^{3}}{\left(y^{3}\right)^{2}}\right]+\frac{1}{2\left(y^{3}\right)^{3}}\left[\left(\dot{x}^{3}\right)^{2}+\left(\dot{y}^{3}\right)^{2}\right]=e^{2 U} \frac{\partial V_{\mathrm{BH}}}{\partial y^{3}} \text {. }
\end{aligned}
$$

Assume the solutions take the following form

$$
\begin{gathered}
e^{-4 U}=4 H^{0} H_{1} H_{2} H_{3}-c^{2}, \\
z^{1}=x^{1}-i \frac{e^{2 U}}{2 H^{0} H_{1}}, \quad z^{2}=x^{2}-i \frac{e^{2 U}}{2 H^{0} H_{2}}, \quad z^{3}=x^{3}-i \frac{e^{2 U}}{2 H^{0} H_{3}}, \\
H^{0}=a^{0}+p^{0} \tau, \quad H_{1}=a_{1}+q_{1} \tau, \quad H_{2}=a_{2}+q_{2} \tau, \quad H_{3}=a_{3}+q_{3} \tau,
\end{gathered}
$$

where $c, a^{0}$ and $a_{i}(\mathrm{i}=1,2,3)$ are numerical constants. Let us solve (3.9) first. From (3.12) we have the following expression

$$
\begin{aligned}
\ddot{U}= & 4 e^{8 U}\left(p^{0} H_{1} H_{2} H_{3}+q_{1} H^{0} H_{2} H_{3}+q_{2} H^{0} H_{1} H_{3}+q_{3} H^{0} H_{1} H_{2}\right)^{2} \\
& -e^{4 U}\left(2 p^{0} q_{1} H_{2} H_{3}+2 p^{0} q_{2} H_{1} H_{3}+2 p^{0} q_{3} H_{1} H_{2}\right. \\
& \left.+2 q_{1} q_{2} H^{0} H_{3}+2 q_{1} q_{3} H^{0} H_{2}+2 q_{2} q_{3} H^{0} H_{1}\right) .
\end{aligned}
$$

Thus we can expand both $\ddot{U}$ and $e^{2 U} V_{\mathrm{BH}}$ using (3.8) and (3.15) then compare the corresponding terms to find the solutions to $x^{1}, x^{2}, x^{3}$. Consider the $q_{1}^{2}$ term for example, we obtain

$$
4 e^{8 U}\left(H^{0} H_{2} H_{3}\right)^{2}=\frac{e^{2 U}}{2 y^{1} y^{2} y^{3}}\left[\left(x^{1}\right)^{2}+\left(y^{1}\right)^{2}\right]
$$

Solving this equation gives

$$
x^{1}=\frac{c}{2 H^{0} H_{1}} .
$$

The solutions to $x^{2}$ and $x^{3}$ can be obtained in a similar way, which gives

$$
x^{2}=\frac{c}{2 H^{0} H_{2}}, \quad x^{3}=\frac{c}{2 H^{0} H_{3}} .
$$

One can check that the above solutions solve (3.9) completely.

The next task is to check if the above solutions satisfy the remaining equations of motion and the constraint. Due to the cyclic symmetries of $x^{i}$ and $y^{i}$ appear in $V_{\mathrm{BH}}$, it is necessary to check the first two equations in (3.10) and (3.11). The left hand side of the first equation in (3.10) gives

$$
\frac{1}{2} \frac{d}{d \tau}\left[\frac{\dot{x}^{1}}{\left(y^{1}\right)^{2}}\right]=-2 c p^{0} q_{1} e^{4 U}-4 c\left(p^{0} H_{1}+q_{1} H^{0}\right) e^{4 U} \dot{U} .
$$

Note that

$$
\dot{U}=-e^{4 U}\left(p^{0} H_{1} H_{2} H_{3}+q_{1} H^{0} H_{2} H_{3}+q_{2} H^{0} H_{1} H_{3}+q_{3} H^{0} H_{1} H_{2}\right)
$$


and

$$
\begin{aligned}
\frac{\partial V_{\mathrm{BH}}}{\partial x^{1}}= & \frac{1}{2 y^{1} y^{2} y^{3}}\left\{2 q_{1}^{2}+2 q_{1} q_{2} x_{2}+2 q_{1} q_{3} x_{3}+2\left(p^{0}\right)^{2} x^{1}\left[\left(x^{2}\right)^{2}+\left(y^{2}\right)^{2}\right]\left[\left(x^{3}\right)^{2}+\left(y^{3}\right)^{2}\right]\right. \\
& \left.+4 p^{0} q_{1} x^{1} x^{2} x^{3}+2 p^{0} q_{2}\left[\left(x^{2}\right)^{2}+\left(y^{2}\right)^{2}\right] x^{3}+2 p^{0} q_{3}\left[\left(x^{3}\right)^{2}+\left(y^{3}\right)^{2}\right] x^{2}\right\}
\end{aligned}
$$

Substituting the expressions of $x^{1}$ and $y^{1}$ to the above equations, one can find that both sides match after a lengthy calculation.

Subsequently we rewrite the first equation of (3.11) as follows:

$$
\frac{1}{2} \frac{\ddot{y}^{1}}{\left(y^{1}\right)^{2}}+\frac{1}{2\left(y^{1}\right)^{3}}\left(\dot{x}^{1}\right)^{2}-\frac{1}{2\left(y^{1}\right)^{3}}\left(\dot{y}^{1}\right)^{2}=e^{2 U} \frac{\partial V_{\mathrm{BH}}}{\partial y^{1}} .
$$

Note that

$$
\begin{gathered}
\dot{x}^{1}=-x^{1}\left(\frac{p^{0}}{H^{0}}+\frac{q_{1}}{H_{1}}\right), \\
\dot{y}^{1}=-y^{1}\left(2 \dot{U}+\frac{p^{0}}{H^{0}}+\frac{q_{1}}{H_{1}}\right), \\
\ddot{y}^{1}=y^{1}\left[4 \dot{U}^{2}+4 \dot{U}\left(\frac{p^{0}}{H^{0}}+\frac{q_{1}}{H_{1}}\right)+\left(\frac{p^{0}}{H^{0}}+\frac{q_{1}}{H_{1}}\right)^{2}-2 \ddot{U}+\frac{\left(p^{0}\right)^{2}}{\left(H^{0}\right)^{2}}+\frac{q_{1}^{2}}{H_{1}^{2}}\right] .
\end{gathered}
$$

Thus the left hand side of (3.22) can be given as

$$
\frac{1}{2} \frac{\ddot{y}^{1}}{\left(y^{1}\right)^{2}}+\frac{1}{2\left(y^{1}\right)^{3}}\left(\dot{x}^{1}\right)^{2}-\frac{1}{2\left(y^{1}\right)^{3}}\left(\dot{y}^{1}\right)^{2}=-\frac{\ddot{U}}{y^{1}}+\frac{1}{2 y^{1}}\left[\frac{\left(p^{0}\right)^{2}}{\left(H^{0}\right)^{2}}+\frac{q_{1}^{2}}{H_{1}^{2}}\right]+\frac{\left(x^{1}\right)^{2}}{2\left(y^{1}\right)^{3}}\left(\frac{p^{0}}{H^{0}}+\frac{q_{1}}{H_{1}}\right)^{2}
$$

while

$$
\frac{\partial V_{\mathrm{BH}}}{\partial y^{1}}=-\frac{V_{\mathrm{BH}}}{y^{1}}+\frac{1}{2 y^{1} y^{2} y^{3}}\left\{2 q_{1}^{2} y^{1}+2\left(p^{0}\right)^{2} y^{1}\left[\left(x^{2}\right)^{2}+\left(y^{2}\right)^{2}\right]\left[\left(x^{3}\right)^{2}+\left(y^{3}\right)^{2}\right]+4 p^{0} q_{1} x^{2} x^{3} y^{1}\right\} .
$$

One can find that our solutions also satisfy this equation by making use of the equation of motion (3.9).

Finally, we have to check the constraint (2.17), which can be rewritten as follows

$$
(\dot{U})^{2}+\frac{1}{4} \sum_{a=1}^{3} \frac{\left(\dot{x}^{a}\right)^{2}+\left(\dot{y}^{a}\right)^{2}}{\left(y^{a}\right)^{2}}=e^{2 U} V_{\mathrm{BH}}
$$

By making use of (3.20), (3.23), (3.24) as well as the cyclic permutations of the last two equations, one can find that the left hand side of (3.28) can be simplified dramatically,

$$
(\dot{U})^{2}+\frac{1}{4} \sum_{a=1}^{3} \frac{\left(\dot{x}^{a}\right)^{2}+\left(\dot{y}^{a}\right)^{2}}{\left(y^{a}\right)^{2}}=\ddot{U}
$$

Thus the constraint is also satisfied according to the equation of motion (3.9). 
Now we would like to summarize our main result. The solution to the non-BPS D2-D6 system can be expressed as

$$
\begin{gathered}
e^{-4 U}=4 H^{0} H_{1} H_{2} H_{3}-c^{2}, \\
z^{1}=\frac{c}{2 H^{0} H_{1}}-i \frac{e^{2 U}}{2 H^{0} H_{1}}, \quad z^{2}=\frac{c}{2 H^{0} H_{2}}-i \frac{e^{2 U}}{2 H^{0} H_{2}}, \quad z^{3}=\frac{c}{2 H^{0} H_{3}}-i \frac{e^{2 U}}{2 H^{0} H_{3}}, \\
H^{0}=a^{0}+p^{0} \tau, \quad H_{1}=a_{1}+q_{1} \tau, \quad H_{2}=a_{2}+q_{2} \tau, \quad H_{3}=a_{3}+q_{3} \tau .
\end{gathered}
$$

It can be easily seen that the above solutions have a manifestly dual form with respect to the solution obtained in [15]. The numerical constants are left undetermined due to subtle

points which will be discussed in the last section. One can see that the moduli exhibit the same attractor values at the horizon as those of the simple D2-D6 non-BPS black hole. The entropy is given by

$$
S=\left.\pi V\right|_{e x t}=2 \pi \sqrt{p^{0} q_{1} q_{2} q_{3}}
$$

which is also the same as that of the simple D2-D6 case.

\section{Adding D2 Charges to D0-D4 System}

In this section we obtain new solutions carrying D0-D2-D4 charges by transforming the original D0-D4 solution in [15] under $S L(2, \mathbf{Z})^{3}$ duality.

\subsection{U-duality for STU black holes}

The symplectic structure of $\mathcal{N}=2$ supergravity admits a symplectic invariant $I_{1}$, which is given by

$$
I_{1}=|Z|^{2}+|D Z|^{2}
$$

$I_{1}$ becomes a function of charges when restricted to STU model, which is given by $I_{1}=$ $\sqrt{|\mathcal{W}(\Gamma)|}$, where

$$
\begin{aligned}
\mathcal{W}(\Gamma)= & 4\left(\left(p^{1} q_{1}\right)\left(p^{2} q_{2}\right)+\left(p^{1} q_{1}\right)\left(p^{3} q_{3}\right)+\left(p^{2} q_{2}\right)\left(p^{3} q_{3}\right)\right) \\
& -\left(p^{\Lambda} q_{\Lambda}\right)^{2}-4 p^{0} q_{1} q_{2} q_{3}+4 q_{0} p^{1} p^{2} p^{3}
\end{aligned}
$$

and $\Gamma=\left(p^{\Lambda} q_{\Lambda}\right), \Lambda=0,1,2,3$. The symplectic invariance of special geometry ensures that the Lagrangian has an $S p(8, \mathbf{Z})$ symmetry, which reduces to $[S L(2, \mathbf{Z})]^{3}$ at the level of the the equations of motion. Given an $S L(2, \mathbf{Z})$ matrix

$$
\left(\begin{array}{ll}
a & b \\
c & d
\end{array}\right)
$$

with $a d-b c=1$, the moduli change as

$$
\tilde{z}^{a}=\frac{a z^{a}+b}{c z^{a}+d}
$$


In [6], the authors changed the charges appeared in the attractor values of the moduli to the corresponding harmonic functions. Consider the spherically symmetric, static metric ansatz:

$$
d s^{2}=-e^{2 U} d t^{2}+e^{-2 U} d \vec{x}^{2},
$$

where the metric is given by

$$
e^{-2 U}=\sqrt{|\mathcal{W}(\mathbf{H})|} .
$$

In order to obtain general (D0, D2, D4, D6) system from their D2-D6 solution, they took a specific element of $[S L(2, \mathbf{Z})]^{3}$ and obtained the general solutions via duality transformations. We will find the D0-D2-D4 solution in a similar way in the next section.

\subsection{The D0-D2-D4 solution}

First consider the general superpotential (2.6). For a configuration carrying D0-D4 charges

$$
W=q_{0}-3 D_{A B} z^{A} z^{B},
$$

where $D_{A B} \equiv D_{A B C} p^{C}$. If we add D2 charges to the system, the superpotential turns out to be

$$
W=q_{0}+q_{A} z^{A}-3 D_{A B} z^{A} z^{B},
$$

However, if we do the following transformations

$$
\hat{q}_{0}=q_{0}+\frac{1}{12} D^{A B} q_{A} q_{B}, \quad \hat{z}^{A}=z^{A}-\frac{1}{6} D^{A B} q_{B},
$$

where $D^{A B} \equiv\left(D_{A B}\right)^{-1}$. Then the D0-D2-D4 superpotential becomes

$$
W=\hat{q}_{0}-3 D_{A B} \hat{z}^{A} \hat{z}^{B},
$$

which has the same form as the D0-D4 case.

Now let us specialize to the STU model. The matrices $D_{a b}$ and $D^{a b}$ are given explicitly as follows:

$$
D_{a b}=\frac{1}{6}\left(\begin{array}{ccc}
0 & p^{3} & p^{2} \\
p^{3} & 0 & p^{1} \\
p^{2} & p^{1} & 0
\end{array}\right)
$$

The inverse matrix

$$
D^{a b}=\left(\begin{array}{ccc}
-\frac{3 p^{1}}{p^{2} p^{3}} & \frac{3}{p^{3}} & \frac{3}{p^{2}} \\
\frac{3}{p^{3}} & -\frac{3 p^{2}}{p^{1} p^{3}} & \frac{3}{p^{1}} \\
\frac{3}{p^{2}} & \frac{3}{p^{1}} & -\frac{3 p^{3}}{p^{1} p^{2}}
\end{array}\right)
$$

Then from (4.8) we have the following transformations

$$
z^{\prime a}=z^{a}+k^{a},
$$


where the quantities with primes belong to the D0-D2-D4 system and the quantities without primes belong to the original D0-D4 system from now on. $k^{a}$ can be written as

$$
\begin{aligned}
k^{1} & \equiv \frac{1}{6} D^{1 b} q_{b}=\frac{q_{2}}{2 p^{3}}+\frac{q_{3}}{2 p^{2}}-\frac{p^{1} q_{1}}{2 p^{2} p^{3}}, \\
k^{2} & \equiv \frac{1}{6} D^{2 b} q_{b}=\frac{q_{1}}{2 p^{3}}+\frac{q_{3}}{2 p^{1}}-\frac{p^{2} q_{2}}{2 p^{1} p^{3}}, \\
k^{3} & \equiv \frac{1}{6} D^{3 b} q_{b}=\frac{q_{1}}{2 p^{2}}+\frac{q_{2}}{2 p^{1}}-\frac{p^{3} q_{3}}{2 p^{1} p^{2}} .
\end{aligned}
$$

Note that the charge configuration of the D0-D4 system is expressed as $\left(\hat{q}_{0}, 0,0, p^{a}\right)$ while for the D0-D2-D4 system we have $\left(q_{0}, q_{a}, 0, p^{\prime a}\right)$.

According to (4.3) and (4.12), we can write down the $[S L(2, \mathbf{Z})]^{3}$ matrices

$$
M_{1}=\left(\begin{array}{cc}
1 & k^{1} \\
0 & 1
\end{array}\right), \quad M_{2}=\left(\begin{array}{cc}
1 & k^{2} \\
0 & 1
\end{array}\right), \quad M_{3}=\left(\begin{array}{cc}
1 & k^{3} \\
0 & 1
\end{array}\right) .
$$

Our task is to generalize new solutions by making use of these $[S L(2, \mathbf{Z})]^{3}$ matrices.

Take the same notations as those in [15]

$$
\begin{aligned}
& p^{0}=a_{111}, \quad q_{0}=-a_{000}, \quad p^{1}=a_{011}, \quad q_{1}=a_{100}, \\
& p^{2}=a_{101}, \quad q_{2}=a_{010}, \quad p^{3}=a_{110}, \quad q_{3}=a_{001},
\end{aligned}
$$

which transform as

$$
a_{i^{\prime} j^{\prime} k^{\prime}}^{\prime}=\left(M_{1}\right)_{i^{\prime}}{ }^{i}\left(M_{2}\right)_{j^{\prime}}{ }^{j}\left(M_{3}\right)_{k^{\prime}}{ }^{k} a_{i j k} \quad i, j, k=0,1 .
$$

Using (4.14), one can check that

$$
\left(\hat{q_{0}}, 0,0, p^{a}\right) \Rightarrow\left(q_{0}, q_{a}, 0, p^{a}\right) .
$$

Similarly, the harmonic functions transform as

$$
\begin{gathered}
H^{\prime 1}=H^{1}, \quad H^{\prime 2}=H^{2}, \quad H^{\prime 3}=H^{3} . \\
H_{1}^{\prime}=k^{3} H^{2}+k^{2} H^{3}=\frac{1}{\sqrt{2}}\left(k^{2}+k^{3}\right)+q_{1} \tau, \\
H_{2}^{\prime}=k^{3} H^{1}+k^{1} H^{3}=\frac{1}{\sqrt{2}}\left(k^{1}+k^{3}\right)+q_{2} \tau, \\
H_{3}^{\prime}=k^{1} H^{2}+k^{2} H^{1}=\frac{1}{\sqrt{2}}\left(k^{1}+k^{2}\right)+q_{3} \tau, \\
H_{0}^{\prime}=H_{0}-k^{1} k^{2} H^{3}+k^{1} k^{3} H^{2}+k^{2} k^{3} H^{1} \\
=\frac{1}{\sqrt{2}}\left[\left(1+B^{2}\right)-\left(k^{1} k^{2}+k^{2} k^{3}+k^{1} k^{3}\right)\right]+q_{0} \tau .
\end{gathered}
$$


We can see that the duality invariant does not change indeed

$$
\begin{aligned}
I_{1}(\Gamma)= & 4 \hat{q}_{0} p^{1} p^{2} p^{3} \\
= & 4 q_{0} p^{1} p^{2} p^{3}-\left(p^{1}\right)^{2} q_{1}^{2}-\left(p^{2}\right)^{2} q_{2}^{2}-\left(p^{3}\right)^{2} q_{3}^{2} \\
& +2 q_{1} q_{2} p^{1} p^{2}+2 q_{1} q_{3} p^{1} p^{3}+2 q_{2} q_{3} p^{2} p^{3} . \\
I_{1}^{\prime}(\Gamma)= & 4 q_{0} p^{1} p^{2} p^{3}-\left(p^{1} q_{1}+p^{2} q_{2}+p^{3} q_{3}\right)^{2}+4\left(p^{1} q_{1} p^{2} q_{2}+p^{1} q_{1} p^{3} q_{3}+p^{2} q_{2} p^{3} q_{3}\right) \\
= & 4 q_{0} p^{1} p^{2} p^{3}-\left(p^{1}\right)^{2} q_{1}^{2}-\left(p^{2}\right)^{2} q_{2}^{2}-\left(p^{3}\right)^{2} q_{3}^{2} \\
& +2 q_{1} q_{2} p^{1} p^{2}+2 q_{1} q_{3} p^{1} p^{3}+2 q_{2} q_{3} p^{2} p^{3} \\
= & I_{1}
\end{aligned}
$$

Furthermore, we can see that $\mathcal{W}(\mathbf{H})$ is also invariant.

$$
\begin{gathered}
\mathcal{W}(\mathbf{H})=4 H_{0} H^{1} H^{2} H^{3} . \\
\mathcal{W}^{\prime}\left(\mathbf{H}^{\prime}\right)=4\left(\left(H^{\prime 1} H_{1}^{\prime}\right)\left(H^{\prime 2} H_{2}^{\prime}\right)+\left(H^{\prime 1} H_{1}^{\prime}\right)\left(H^{\prime 3} H_{3}^{\prime}\right)+\left(H^{\prime 2} H_{2}^{\prime}\right)\left(H^{\prime 3} H_{3}^{\prime}\right)\right) \\
-\left(H^{\prime 1} H_{1}^{\prime}+H^{\prime 2} H_{2}^{\prime}+H^{\prime 3} H_{3}^{\prime}\right)^{2}+4 H_{0}^{\prime} H^{\prime 1} H^{\prime 2} H^{\prime 3} .
\end{gathered}
$$

After substituting the expressions for $H^{\prime}$ harmonic functions (4.18)- (4.20), one can find that

$$
\mathcal{W}^{\prime}\left(\mathbf{H}^{\prime}\right)=\mathcal{W}(\mathbf{H})
$$

Here the metric is given by

$$
e^{-4 U}=|\mathcal{W}(\mathbf{H})|-c^{2} .
$$

Thus

$$
e^{-2 U^{\prime}}=e^{-2 U} .
$$

Now we have to check whether the new solution satisfies the equations of motion. From (4.12) and (4.27) we can see that the left hand side of the equations of motion and the constraint remain unchanged. Thus we just need to check if the effective potential $V_{\mathrm{BH}}$ on the right hand side remains invariant. It can be easily seen that

$$
K^{\prime}=K, \quad W=W^{\prime}, \quad D_{a} Z=D_{a \prime} Z^{\prime},
$$

after taking all the relevant formulae into account. Thus by the definition of $V_{\mathrm{BH}}$, we have

$$
V_{\mathrm{BH}}^{\prime}=V_{\mathrm{BH}} .
$$

Then our new solution also satisfies the equations of motion and the constraint.

One can easily obtain the attractor values of the moduli

$$
z^{a}=k^{a}-i \sqrt{\frac{-2 q_{0} p^{a}}{s_{a b c} p^{b} p^{c}}},
$$

which is the same as those discussed in previous examples. The entropy is given by

$$
S_{\mathrm{BH}}=\left.\pi V_{\mathrm{BH}}\right|_{e x t}=2 \pi \sqrt{\hat{q}_{0} p^{1} p^{2} p^{3}},
$$

which also agrees with the previously known D0-D2-D4 entropy. 


\section{Summary and Discussion}

In this note we obtain the non-BPS, extremal, spherically symmetric black hole solutions of four-dimensional supergravity, carrying D2-D6 and D0-D2-D4 charges. The D2-D6 solution contains general complex moduli and is manifestly dual to the D0-D4 cousin appeared in [15]. The D0-D2-D4 solution is obtained by $[S L(2, \mathbf{Z})]^{3}$ duality transformations from the D0-D4 solution. Both of our solutions give the same attractor values of the moduli and the same entropies as those of previously known examples carrying the same charges. One may obtain new solutions carrying general (D0, D2,D4,D6) charges from the known solutions by duality transformations.

One subtle point is the determination of the numerical constants in the D2-D6 solution. Of course one can take the same values as those in [15], that is,

$$
c=B, \quad, a_{0}=\frac{1}{\sqrt{2}}\left(1+B^{2}\right), \quad a^{1}=a^{2}=a^{3}=\frac{1}{\sqrt{2}} .
$$

Thus one can obtain the mass by expanding the warp factor,

$$
2 G_{N} M_{\mathrm{non}-\mathrm{BPS}}=\frac{1}{\sqrt{2}}\left(\left|p^{0}\right|+\sum_{a} q_{a}\left(1+B^{2}\right)\right),
$$

which has a similar form as that given in [15]. This can be interpreted as the sum of the masses of the D2 and D6-branes, which also exhibits a marginal bound state behavior. However, in such cases the asymptotic values of the moduli are different,

$$
z^{a}=\frac{B-i}{1+B^{2}}
$$

which means that the normalization of the asymptotic moduli should be different from that in [15].

Another interesting non-BPS configuration is the D0-D6 system, which has been extensively studied in recent years, see e.g. [17] [18] [19] [20]. The attractor mechanism for D0-D6 Kaluza-Klein black holes has been discussed in [21] using the entropy function formalism. Since our solution is manifest dual to the D0-D4 solution, it will be interesting to study the relations between our solution and the D0-D6 system discussed in [15].

The STU model has been discussed in [22] in another interesting way, that is, such a model can be tackled in the context of quantum information theory. The use of this formalism expresses the black hole potential in an especially elegant form as the norm squared of a suitable tripartite entangled state. Then the classification of solutions proceeds with analysing the charge codes using some elements of quantum error correction. However,

only doubly extremal solutions were discussed in that paper for illustration. So it would be interesting to extend similar analysis using the more general solutions.

A further direction is to generalize the famous "OSV" conjecture [23], which relates the partition function of BPS black holes to the partition function of topological strings, to non-BPS case. In a recent paper [24], Sarakin and Vafa pointed out that there was some 
subtle points when generalizing the original "OSV" conjecture to non-BPS cases. Thus an extension of OSV that can be applied simultaneously to both BPS and non-BPS black holes is needed, which is more difficult to realize. However, the various exact solutions of non-BPS black holes provide concrete examples for testing their conjecture. We would like to study this problem in the near future.

Note Added: After the first version appearing on arXiv, we were informed with [16]. In that interesting paper the authors constructed interpolating solutions describing singlecenter static extremal non-supersymetric black holes in four dimensional $\mathcal{N}=2$ supergravity with cubic prepotentials. They derived and solved the first-order flow equations for 5D rotating electrically charged extremal black holes in a Taub-NUT geometry. Then using the $4 \mathrm{D} / 5 \mathrm{D}$ connections they obtained the corresponding 4D solutions. One key point for these results was that the 5D geometry was assumed to be a time fibration over a HyperKähler base. When the 4D prepotential contains a cubic term, the corresponding solutions to the first-order flow equations are

$$
\begin{gathered}
e^{-4 U}=\frac{4}{9} N\left(H_{A} f^{-1 / 2} X^{A}\right)^{2}-c^{2}, \\
z^{A}=\frac{3}{2}\left(\frac{c+i e^{-2 U}}{N H_{B} f^{-1 / 2} X^{B}}\right) f^{-1 / 2} X^{A} .
\end{gathered}
$$

One can find that our D2-D6 solution agrees with their solution when restricted to STU mode 1 .

\section{Acknowledgements}

We thank G. Cardoso and P. Levay for correspondence. The work was supported in part by a grant from Chinese Academy of Sciences, by NSFC under grants No. 10325525 and No. 90403029.

\section{References}

[1] S. Ferrara, R. Kallosh and A. Strominger, "N=2 extremal black holes," Phys. Rev. D 52, 5412 (1995) arXiv:hep-th/9508072].

A. Strominger, "Macroscopic Entropy of $N=2$ Extremal Black Holes," Phys. Lett. B 383, 39 (1996) arXiv:hep-th/9602111.

S. Ferrara and R. Kallosh, "Supersymmetry and Attractors," Phys. Rev. D 54, 1514 (1996) arXiv:hep-th/9602136.

S. Ferrara and R. Kallosh, "Universality of Supersymmetric Attractors," Phys. Rev. D 54, 1525 (1996) arXiv:hep-th/9603090.

[2] G. Lopes Cardoso, B. de Wit and T. Mohaupt, "Corrections to macroscopic supersymmetric black-hole entropy," Phys. Lett. B 451, 309 (1999) arXiv:hep-th/9812082.

G. Lopes Cardoso, B. de Wit and T. Mohaupt, "Deviations from the area law for

\footnotetext{
${ }^{1}$ We thank G. Cardoso for pointing this to us.
} 
supersymmetric black holes," Fortsch. Phys. 48, 49 (2000) arXiv:hep-th/9904005. G. Lopes Cardoso, B. de Wit and T. Mohaupt, "Macroscopic entropy formulae and non-holomorphic corrections for supersymmetric black holes," Nucl. Phys. B 567, 87 (2000) arXiv:hep-th/9906094.

G. Lopes Cardoso, B. de Wit, J. Kappeli and T. Mohaupt, "Stationary BPS solutions in $\mathrm{N}=2$ supergravity with $\mathrm{R}^{* *} 2$ interactions," JHEP 0012, 019 (2000) arXiv:hep-th/0009234.

[3] S. Ferrara, G. W. Gibbons and R. Kallosh, "Black holes and critical points in moduli space," Nucl. Phys. B 500, 75 (1997) arXiv:hep-th/9702103.

[4] K. Goldstein, N. Iizuka, R. P. Jena and S. P. Trivedi, "Non-supersymmetric attractors," Phys. Rev. D 72, 124021 (2005) arXiv:hep-th/0507096.

P. K. Tripathy and S. P. Trivedi, "Non-supersymmetric attractors in string theory," JHEP 0603, 022 (2006) arXiv:hep-th/0511117.

K. Goldstein, R. P. Jena, G. Mandal and S. P. Trivedi, "A C-function for nonsupersymmetric attractors," JHEP 0602, 053 (2006) arXiv:hep-th/0512138].

[5] R. Kallosh, N. Sivanandam and M. Soroush, "The non-BPS black hole attractor equation," JHEP 0603, 060 (2006) arXiv:hep-th/0602005].

[6] R. Kallosh, N. Sivanandam and M. Soroush, "Exact attractive non-BPS STU black holes," Phys. Rev. D 74, 065008 (2006) arXiv:hep-th/0606263].

[7] A. Dabholkar, A. Sen and S. P. Trivedi, "Black hole microstates and attractor without supersymmetry," arXiv:hep-th/0611143.

[8] M. Alishahiha and H. Ebrahim, "Non-supersymmetric attractors and entropy function," JHEP 0603, 003 (2006) arXiv:hep-th/0601016].

B. Chandrasekhar, S. Parvizi, A. Tavanfar and H. Yavartanoo, "Non-supersymmetric attractors in R**2 gravities," JHEP 0608, 004 (2006) arXiv:hep-th/0602022.

S. Parvizi and A. Tavanfar, "Partition function of non-supersymmetric black holes in the supergravity limit," arXiv:hep-th/0602292.

R. Kallosh, "From BPS to non-BPS black holes canonically," arXiv:hep-th/0603003.

B. Sahoo and A. Sen, "Higher derivative corrections to non-supersymmetric extremal black holes in N = 2 supergravity," JHEP 0609, 029 (2006) arXiv:hep-th/0603149.

G. L. Cardoso, V. Grass, D. Lust and J. Perz, "Extremal non-BPS black holes and entropy extremization," JHEP 0609, 078 (2006) arXiv:hep-th/0607202].

H. Arfaei and R. Fareghbal, "Double-horizon limit and decoupling of the dynamics at the horizon," JHEP 0701, 060 (2007) arXiv:hep-th/0608222.

D. Astefanesei, K. Goldstein and S. Mahapatra, "Moduli and (un)attractor black hole thermodynamics," arXiv:hep-th/0611140.

B. Chandrasekhar, H. Yavartanoo and S. Yun, "Non-Supersymmetric Attractors in BI black holes," arXiv:hep-th/0611240. 
C. M. Chen, D. V. Gal'tsov and D. G. Orlov, "Extremal black holes in D = 4 GaussBonnet gravity," Phys. Rev. D 75, 084030 (2007) |arXiv:hep-th/0701004|.

R. D'Auria, S. Ferrara and M. Trigiante, "Critical points of the black-hole potential for homogeneous special geometries," JHEP 0703, 097 (2007) |arXiv:hep-th/0701090. A. Ceresole and G. Dall'Agata, "Flow equations for non-BPS extremal black holes," JHEP 0703, 110 (2007) [arXiv:hep-th/0702088].

R. G. Cai and L. M. Cao, "On the Entropy Function and the Attractor Mechanism for Spherically Symmetric Extremal Black Holes," Phys. Rev. D 76, 064010 (2007) arXiv:0704.1239 [hep-th]].

S. Ferrara and A. Marrani, "N=8 non-BPS Attractors, Fixed Scalars and Magic Supergravities," Nucl. Phys. B 788, 63 (2008) [arXiv:0705.3866 [hep-th]].

S. Nampuri, P. K. Tripathy and S. P. Trivedi, "On The Stability of Non-Supersymmetric Attractors in String Theory," JHEP 0708, 054 (2007) arXiv:0705.4554 [hep-th]].

S. Ferrara and A. Marrani, "On the Moduli Space of non-BPS Attractors for $\mathrm{N}=2$ Symmetric Manifolds," Phys. Lett. B 652, 111 (2007) [arXiv:0706.1667 [hep-th]].

D. Astefanesei and H. Yavartanoo, "Stationary black holes and attractor mechanism," arXiv:0706.1847 [hep-th].

S. Yun, "Non-Supersymmetric Unattractors in Born-Infeld Black Holes," arXiv:0706.2046 [hep-th].

A. Ceresole, S. Ferrara and A. Marrani, " $4 \mathrm{~d} / 5 \mathrm{~d}$ Correspondence for the Black Hole Potential and its Critical Points," arXiv:0707.0964 [hep-th].

M. M. Anber and D. Kastor, "The Attractor Mechanism in Gauss-Bonnet Gravity," JHEP 0710, 084 (2007) arXiv:0707.1464 [hep-th]].

Y. S. Myung, Y. W. Kim and Y. J. Park, "New attractor mechanism for spherically symmetric extremal black holes," arXiv:0707.1933 [hep-th].

S. Bellucci, A. Marrani, E. Orazi and A. Shcherbakov, "Attractors with Vanishing Central Charge," Phys. Lett. B 655, 185 (2007) arXiv:0707.2730 [hep-th]].

H. Arfaei and R. Fareghbal, "Double Horizon Limit, AdS Geometry and Entropy Function," arXiv:0708.0240 [hep-th].

X. Gao, "Non-supersymmetric Attractors in Born-Infeld Black Holes with a Cosmological Constant," JHEP 0711, 006 (2007) arXiv:0708.1226 [hep-th]].

L. Andrianopoli, S. Ferrara, A. Marrani and M. Trigiante, "Non-BPS Attractors in 5d and 6d Extended Supergravity," arXiv:0709.3488 [hep-th].

D. Gaiotto, W. W. Li and M. Padi, "Non-Supersymmetric Attractor Flow in Symmetric Spaces," arXiv:0710.1638 [hep-th].

S. Bellucci, S. Ferrara, A. Marrani and A. Shcherbakov, "Splitting of Attractors in 1-modulus Quantum Corrected Special Geometry," arXiv:0710.3559 [hep-th].

D. Astefanesei, H. Nastase, H. Yavartanoo and S. Yun, "Moduli flow and nonsupersymmetric AdS attractors," arXiv:0711.0036 [hep-th].

[9] T. Mohaupt, "Black hole entropy, special geometry and strings," Fortsch. Phys. 49, 3 (2001) arXiv:hep-th/0007195. 
B. Pioline, "Lectures on on black holes, topological strings and quantum attractors," Class. Quant. Grav. 23, S981 (2006) arXiv:hep-th/0607227].

F. Larsen, "The attractor mechanism in five dimensions," arXiv:hep-th/0608191.

L. Andrianopoli, R. D'Auria, S. Ferrara and M. Trigiante, "Extremal black holes in supergravity," arXiv:hep-th/0611345.

T. Mohaupt, "Supersymmetric black holes in string theory," Fortsch. Phys. 55, 519 (2007) arXiv:hep-th/0703035.

S. Bellucci, S. Ferrara, R. Kallosh and A. Marrani, "Extremal Black Hole and Flux Vacua Attractors," arXiv:0711.4547 [hep-th].

[10] W. A. Sabra, "General static N = 2 black holes," Mod. Phys. Lett. A 12, 2585 (1997) arXiv:hep-th/9703101.

[11] W. A. Sabra, "Black holes in $\mathrm{N}=2$ supergravity theories and harmonic functions," Nucl. Phys. B 510, 247 (1998) arXiv:hep-th/9704147.

[12] K. Behrndt, D. Lust and W. A. Sabra, "Stationary solutions of N = 2 supergravity," Nucl. Phys. B 510, 264 (1998) arXiv:hep-th/9705169.

[13] B. Bates and F. Denef, "Exact solutions for supersymmetric stationary black hole composites," arXiv:hep-th/0304094.

[14] K. Hotta and T. Kubota, "Exact Solutions and the Attractor Mechanism in Non-BPS Black Holes," arXiv:0707.4554 [hep-th].

[15] E. G. Gimon, F. Larsen and J. Simon, "Black Holes in Supergravity: the non-BPS Branch," arXiv:0710.4967 [hep-th].

[16] G. Lopes Cardoso, A. Ceresole, G. Dall'Agata, J. M. Oberreuter and J. Perz, "Firstorder flow equations for extremal black holes in very special geometry," JHEP 0710, 063 (2007) [arXiv:0706.3373 [hep-th]].

[17] W. Taylor, "Adhering 0-branes to 6-branes and 8-branes," Nucl. Phys. B 508, 122 (1997) arXiv:hep-th/9705116].

[18] A. Dhar and G. Mandal, "Probing 4-dimensional nonsupersymmetric black holes carrying D0- and D6-brane charges," Nucl. Phys. B 531, 256 (1998) arXiv:hep-th/9803004].

[19] F. Larsen, "Kaluza-Klein black holes in string theory," arXiv:hep-th/0002166.

[20] R. Emparan and A. Maccarrone, "Statistical description of rotating Kaluza-Klein black holes," Phys. Rev. D 75, 084006 (2007) [arXiv:hep-th/0701150].

[21] D. Astefanesei, K. Goldstein, R. P. Jena, A. Sen and S. P. Trivedi, "Rotating attractors," JHEP 0610, 058 (2006) arXiv:hep-th/0606244. 
[22] P. Levay, "A three-qubit interpretation of BPS and non-BPS STU black holes," Phys. Rev. D 76, 106011 (2007) arXiv:0708.2799 [hep-th]].

[23] H. Ooguri, A. Strominger and C. Vafa, "Black hole attractors and the topological string," Phys. Rev. D 70, 106007 (2004) arXiv:hep-th/0405146].

[24] K. Saraikin and C. Vafa, "Non-supersymmetric Black Holes and Topological Strings," arXiv:hep-th/0703214. 